\title{
CHARACTERIZATION OF MAGNETO-OPTICAL MEDIA BY MAGNETIC FORCE MICROSCOPY
}

\section{H.W. VAN KESTEREN, G.J.P. VAN ENGELEN and B.A.J. JACOBS}

\author{
Philips Research \\ P.O. Box 80 000, 5600 JA Eindhoven, The Netherlands
}

\begin{abstract}
The combination of a high resolution and essentially no sample preparation, makes magnetic force microscopy a powerful characterization technique for magnetic storage media. This is illustrated by images of domains recorded on $\mathrm{GdTbFe}$ and $\mathrm{Co} / \mathrm{Pt}$ magneto-optical disks. Scanning force microscopy with magnetic as well as nonmagnetic tips has been used to obtain more insight into the effects of a mild sputter etch of the dielectric layer before the deposition of a $\mathrm{Co} / \mathrm{Pt}$ multilayer. The main effect of the sputter etch is a reduced roughness of the multilayer which results in a lower disk noise.
\end{abstract}

KEYWORDS: MAGNETIC FORCE MICROSCOPY, Co/Pt MULTILAYERS, MO RECORDING

\section{MAGNETIC FORCE MICROSCOPY}

\section{Introduction}

Scanning force microscopy (SFM) has become a widely used technique for high-resolution imaging of a large variety of samples. The basic principle is that the position or oscillation frequency of a cantilever with a sharp tip at its end is determined by the local interaction of the tip and sample. An image is formed by mapping the change in the interaction during a raster scan over the sample surface. Images representing Van der Waals, electrostatic and magnetic forces can be obtained in this way.

The force microscope used to obtain the images presented in this paper operates with an interferometer to detect the vibration of the tip. To obtain magnetic contrast, the tip is coated with a thin magnetic layer. The force gradient acting on the tip affects the phase of the vibration, which is detected and used in a feedback loop to control the object-tip distance such that a constant force gradient is maintained. To ensure an attractive force during imaging, a small voltage is applied between the tip and the object. As a consequence of the resulting electrostatic force gradient, the magnetic force microscopy (MFM) images show a superposition of the magnetic structure and the surface topography.

MFM is a powerful characterization technique for magnetic storage media [1-6], which is of primary interest in view of their technological importance. The advantage of MFM over other imaging techniques stems from the fact that it is the only technique which combines a high resolution with essentially no specimen preparation. The possibility of imaging regular tapes and disks enables one to relate the recording conditions and carrier and noise levels of the read-out signal directly to the magnetic microstructure of the recording medium.

To resolve the smallest features in MFM images of magnetic recording media, high requirements are posed on the force microscope as well as on the picture processing and visualization. The images in this paper are visualized in three dimensions to enhance small features. For instance, small magnetic structures in the deep ' $V$ '-grooves of MO disks or the subdomain structure within a written bit can be more easily recognized in a three-dimensional image with carefully adjusted light sources than in a two-dimensional grey-scale picture.

In the next section several illustrative MFM images are shown of magnetic storage media. Thereafter, we will describe the tip artefacts that sometimes occur in high-resolution images. Resognition of these artefacts is important because a misunderstanding of these effects can easily lead to a wrong interpretation of an image. In the last part of the paper we will discuss the effects of a mild sputter etch of the dielectric layer on the recording properties of a $\mathrm{Co} / \mathrm{Pt}$ disk. SFM images of sputteretched and non-sputter-etched disks were made to obtain more insight into the observed recording properties.

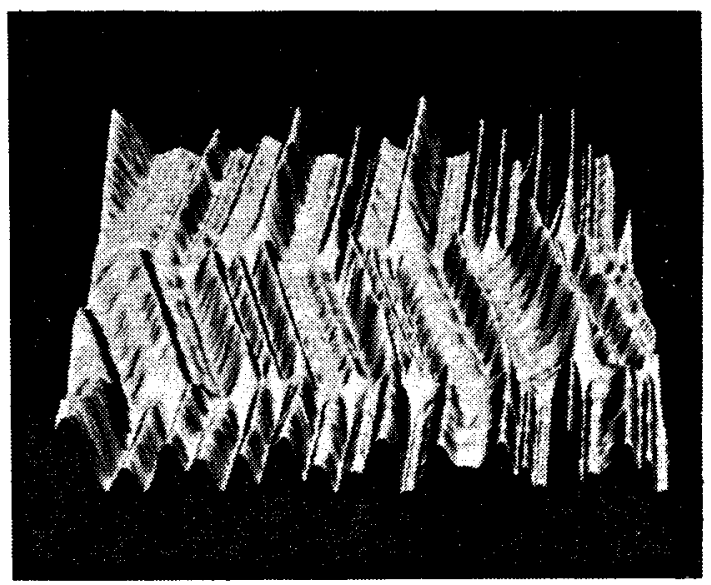

Fig. 1 MFM image of a random bit pattern on an metal-evaporated tape. The tracks have a width of $10 \mu \mathrm{m}$ and the bit size is $0.25 \mu \mathrm{m}$. 
van Kesteren et al.: Characterization of Magneto-Optical Media by Magnetic Force Microscopy

\section{Images of storage media}

Fig. 1 shows an MFM image of bits on an metalevaporated tape. The random bit pattern was recorded with an azimuth on the tape to limit cross-talk between neighbouring tracks. The tracks have a width of $10 \mu \mathrm{m}$ and the bit size is $0.25 \mu \mathrm{m}$. This image illustrates that small bits on longitudinal tape media can be studied very well by MFM. More detailed MFM studies of magnetic recording media can be found for instance in references [1-3].

Figs. $2 \mathrm{a}$ and $\mathrm{b}$ show images of thermomagnetically recorded bits on $\mathrm{MO}$ disks with a $\mathrm{Co} / \mathrm{Pt}$ multilayer film and a GdTbFe film respectively. These images are intended to illustrate the resolutions obtained with MFM on these two media. The deep 'V'-s are the grooves, the rest of the structure is of magnetic origin. These images as well as those following represent areas of about $6.5 \times 6.5 \mathrm{~mm}^{2}$. The $2.5 \mathrm{MHz}$ carriers were written on the disks with laser modulation at $647 \mathrm{~nm}$ using various write-field strengths as indicated in the figure. The linear disk velocity was $5 \mathrm{~m} / \mathrm{s}$ and the carrier and noise levels were detected using a $30 \mathrm{kHz}$ bandwidth.

The image of the domains recorded on a disk with a $\mathrm{Co} / \mathrm{Pt}$ multilayer (fig. $2 \mathrm{a}$ ) shows that for this medium images can be obtained with a resolution better than 50 $\mathrm{nm}$. The domains recorded at 0 and $12.5 \mathrm{kA} / \mathrm{m}$ clearly show the subdomain structure that occurs when the write field is too small, while the domains recorded at $50 \mathrm{kA} / \mathrm{m}$ illustrate the jaggedness of the domain boundary occuring at too high write fields. The jaggedness leads to increased write noise levels, while the presence of subdomains leads to a lower carrier level.

Fig. $2 b$ shows that for GdTbFe the resolution is somewhat lower than for $\mathrm{Co} / \mathrm{Pt}$. However, the images are still sufficiently detailed to reveal important features. The GdTbFe medium had a compensation temperature of about $-40^{\circ} \mathrm{C}$ and was covered by a $20 \mathrm{~nm}$ Al layer. The lower resolution for RE-TM media is a general phenomenon and is due to their ferrimagnetic behaviour and compensation temperature, which is relatively close to room temperature. Another factor which lowers the resolution for RE-TM media is the application of a protection layer and/or a metal layer to enhance the Kerr rotation. As a consequence of the low magnetization at room temperature and the smaller tipsample interaction at larger distances, tips with a relatively thick Co layer had to be used, which resulted in lower resolutions.

The domains recorded on the GdTbFe disk at fields of $12.5 \mathrm{kA} / \mathrm{m}$ and higher have a regular shape, which is also reflected in the almost constant carrier and noise levels. Only the domains recorded at zero field show some jaggedness, which again relates to an increased write noise level. A more extensive discussion of the magnetic domain structure on MO media, as observed by MFM, can be found in [4-6].

In the near future MFM will become important for the characterization of magnetic and $\mathrm{MO}$ recording media with increased storage densities. In optical

\begin{tabular}{ll||c|c|c|c}
$\mathrm{H}_{\text {wr }}$ & $(\mathrm{kA} / \mathrm{m})$ & 50 & 25 & 12.5 & 0 \\
\hline $\mathrm{CNR}$ & $(\mathrm{dB})$ & 45.0 & 48.8 & 48.4 & 46.1 \\
\hline $\mathrm{C}$ & $(\mathrm{dBm})$ & -2.5 & -2.8 & -3.6 & -5.6 \\
\hline $\mathrm{WN}$ & $(\mathrm{dB})$ & 6.8 & 2.7 & 2.3 & 2.6
\end{tabular}

(a)

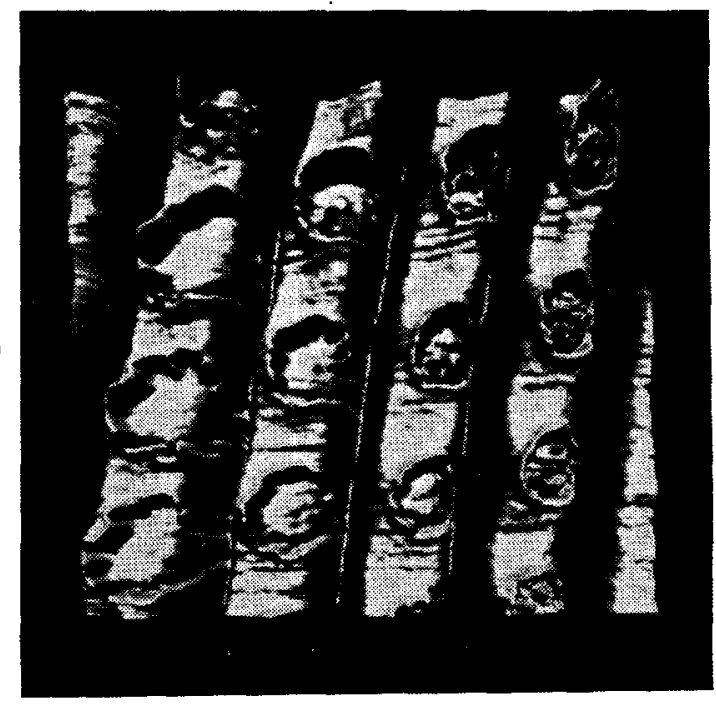

\begin{tabular}{ll||c|c|c|c}
$\mathrm{H}_{\mathrm{wr}}$ & $(\mathrm{lA} / \mathrm{m})$ & 50 & 25 & 12.5 & 0 \\
\hline $\mathrm{CNR}$ & $(\mathrm{dB})$ & 50.5 & 50.5 & 49.2 & 42.0 \\
\hline $\mathrm{C}$ & $(\mathrm{dBm})$ & -3.7 & -4.3 & -4.8 & -7.3 \\
\hline $\mathrm{WN}$ & $(\mathrm{dB})$ & 2.0 & 1.4 & 2.2 & 6.9
\end{tabular}

(b)

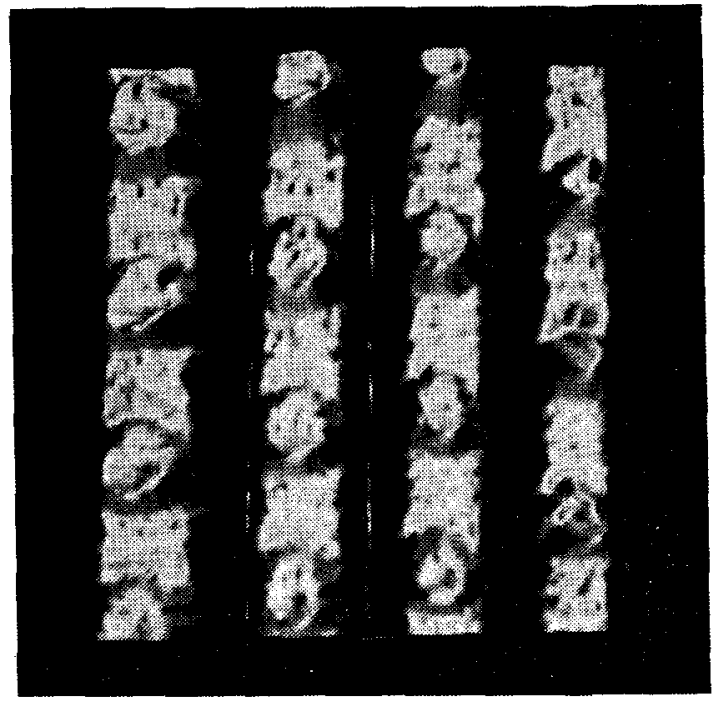

Fig. 2 MFM images of domains recorded at various fields on MO disks with (a) a $14 \times(4 \AA \mathrm{Co}+14 \AA \mathrm{Pt})$ multilayer and (b) a GdTbFe layer. The area is about $6.5 \times 6.5 \mathrm{\mu m}^{2}$. The table above the image gives the write fields for the four tracks as well as the CNR, carrier (C) and write noise (WN) levels of the read-out signal. The order of the columns and the tracks is the same. 
recording, shorter wavelengths enable the detection of smaller domains, which in turn leads to higher storage densities and increased data rates. $\mathrm{Co} / \mathrm{Pt}$ multilayers have the advantageous property that the Kerr rotation almost doubles its value when the wavelength changes from 800 to $400 \mathrm{~nm}$, in contrast to most RE-TM alloys. Fig. 3 shows domains recorded at $458 \mathrm{~nm}$ with laser modulation on a $\mathrm{Co} / \mathrm{Pt}$ multilayer disk. The carrier frequencies were varied and corresponded to domain periods of $5,2,1.25$ and $0.5 \mu \mathrm{m}$. The CNR was $51 \mathrm{~dB}$ for the longest period, which was $3 \mathrm{~dB}$ higher than for a $\mathrm{GdTbFe}$ disk. This was due to the higher Kerr rotation, which led to a higher carrier level. The noise levels of both disks were comparable. The CNR decreased to 21 $\mathrm{dB}$ for the shortest period. The MFM image revealed that even the smallest domains $(0.25 \mu \mathrm{m})$ were well defined. Therefore the loss in CNR should be mainly due to the decrease in the optical transfer function for these very small domains. A more detailed analysis of the recording behaviour of $\mathrm{Co} / \mathrm{Pt}$ at shorter wavelengths can be found in the paper of Zeper et al. [7].

\section{Tip artefacts}

The most critical component of a force microscope is the force sensor. This is due to the fact that the image is a convolution of the structure of the tip and that of the sample. For MFM both the shape and the magnetic structure of the tip are involved. When an image is taken, the microstructure of the tip and object are usually not exactly known, so an ambiguity can exist

\begin{tabular}{ll||c|c|c|c} 
period & $(\mu \mathrm{m})$ & 0.5 & 1.25 & 2 & 5 \\
\hline $\mathrm{CNR}$ & $(\mathrm{dB})$ & 21 & 45 & 49 & 51
\end{tabular}

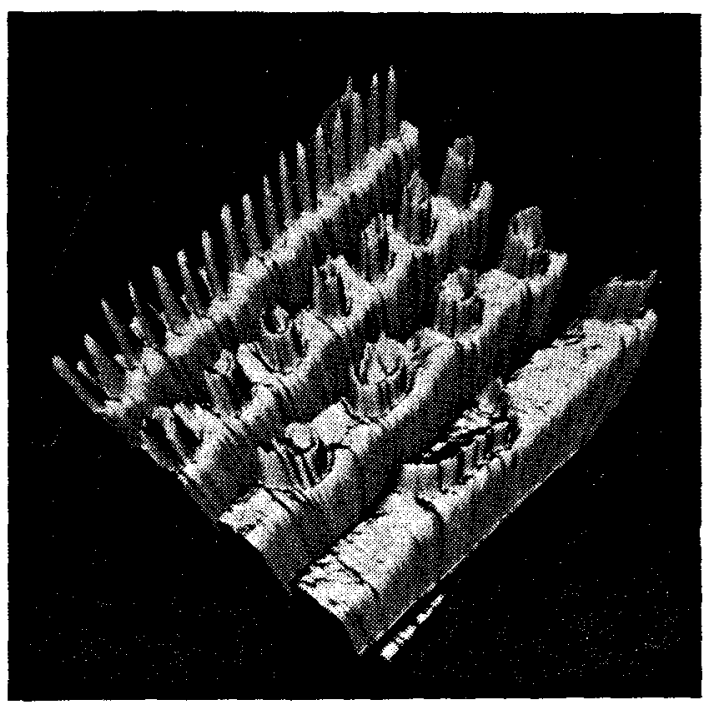

Fig. 3 MFM image of domains recorded at $458 \mathrm{~nm}$ on a disk with a $14 x(4 \AA \mathrm{Co}+14 \AA \mathrm{Pt})$ multilayer. The carrier frequencies were varied and corresponded to the domain periods given in the table. The order of the columns and the tracks is the same. in the interpretation of the image. In extreme cases, the image can even be completely dominated by tip artefacts. The origin of these artefacts lies in inevitable imperfections in the microfabrication of the tips.

We used cantilevers made of thin tungsten wire. A $90^{\circ}$ bend is made manually in the wire with a knife edge. A tip is then etched just beyond the bend and a thin Co layer is galvanically deposited on the tip [8]. These tips can image magnetic domains with a resolution that is better than $50 \mathrm{~nm}$, as is clear from fig. $2 \mathrm{a}$.

In fig. 4 an MFM image is shown of domains recorded on a $\mathrm{Co} / \mathrm{Pt}$ disk at various write fields. The domains seem to be recorded asymmetrically on the land. Furthermore, the small domains in the groove are all situated on a ridge on the right-hand slope of the groove. It was checked by scanning with a non-magnetic tip that there was no ridge on the groove slope. In fact, the shift of the recorded domains out of the center of the land as well as the shift of the small domains out of the groove center are attributed to imaging with a tip on which the magnetic dipole was situated on one of the sides of the tungsten tip. This gave rise to a spatial shift of about $0.1 \mu \mathrm{m}$ between the surface topography image and the magnetic image.

A second tip artefact which is sometimes observed is a superposition of spatially shifted images. An extreme case is shown in fig. 5. On this disk, the domains in the four adjacent tracks were again recorded at various write fields. In the image the domains are situated both on the land and in the groove, and domains recorded at different fields are seen on top of each other. From the physical point of view the latter is very unlikely because the domain recorded last should always dominate. Besides, Kerr microscopy of this disk revealed that domains were not recorded double, nor

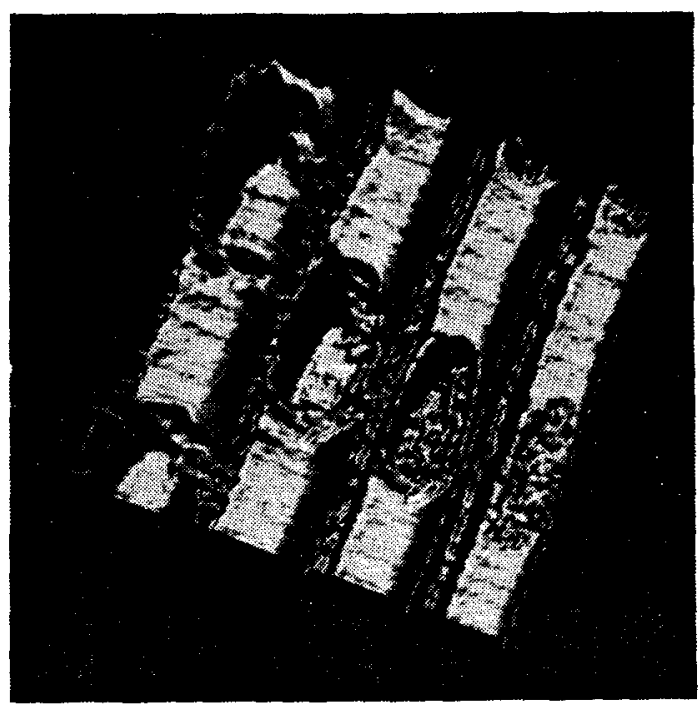

Fig. 4 MFM image of a Co/Pt MO disk showing a spatial shift between the topographic and magnetic structure. This artefact most likely occurs when a tip is used with a magnetic dipole at one of its sides. 
were any domains recorded in the grooves. In fact, these "ghost" images are attributed to a multidomain structure of the tip. Each of these domains acts as a small magnetic tip leading to a superposition of spatially shifted images. Usually, these shifts are in the order of a few tenths of a micron and lead to "double" domain walls and are not as large as several microns as in fig. 5. Imaging with tips with a multidomain structure often gives images with a quite high resolution, as is also evident from fig. 5. This might be due to the fact that these tip domains can be quite small. Apart from a superposition of magnetic images, fig. 5 also shows features of a "ghost" image of the surface structure. On the land an extra structure can be seen, which might reflect a spatially shifted groove structure. This indicates that besides a multidomain structure, this tip probably also had at least two peaks.

Tip artefacts do not only occur for wire cantilevers. Recently, Grütter et al. [9] demonstrated that SFM images made with batch fabricated $\mathrm{Si}_{3} \mathrm{~N}_{4}$ sensors can be dominated by tip artefacts as well. Therefore, the best practice seems to be to image a sample with a wellknown structure to check the tip, before imaging a new sample.

\section{CHARACTERIZATION OF MO DISKS}

The MFM technique can image the domain structure on regular disks. Therefore, the technique is very well suited for studying the effect of the topography of the $2 p$ and dielectric layers on the magnetic microstructure of the MO film. We used SFM to study the effects of a sputter etch of the dielectric layer prior to the deposition of a $\mathrm{Co} / \mathrm{Pt}$ multilayer.

The 3.5" disks studied consisted of a glass substrate,

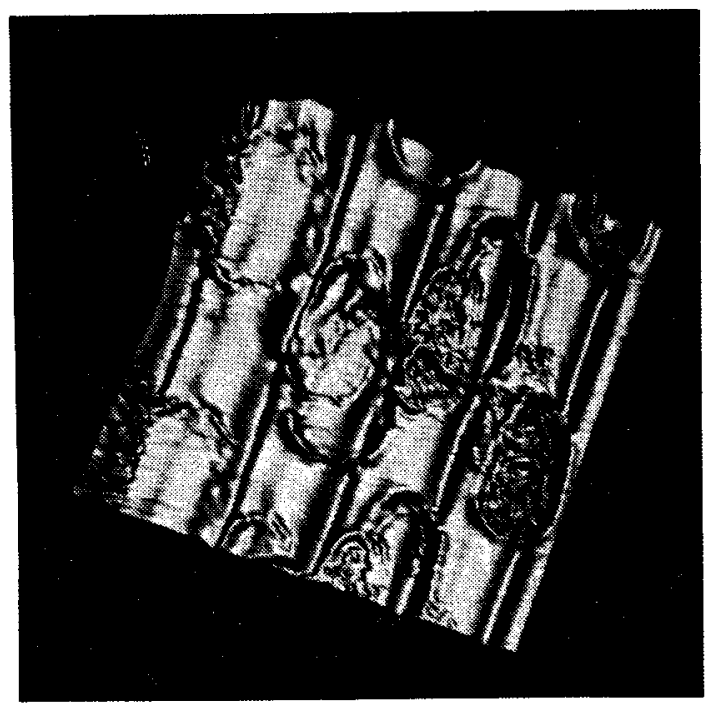

Fig. 5 MFM image of a Co/Pt MO disk showing a superposition of magnetic images. This artefact is most likely due to a tip with a multidomain structure. pregrooved 2p lacquer, a sputtered $A 1 N$ layer and an evaporated $\mathrm{Co} / \mathrm{Pt}$ multilayer. For the surface treatment of the AlN layer, a 10-minute RF sputtering at $200 \mathrm{~W}$ was used. The disks were evaluated at $5 \mathrm{~m} / \mathrm{s}$ in a recorder operating at $647 \mathrm{~nm}$.

The effects of sputter-etching an $\mathrm{AlN}$ or $\mathrm{ZnO}$ surface on the shape of the hysteresis loop of subsequently sputtered Co/Pt multilayers has been discussed by Carcia et al. [10]. Sputter-etching improved the rectangular loop shape of the sputtered multilayers, and the CNRs of the disks increased by several $\mathrm{dB}$. For the sputter-etched and non sputter-etched disk with an evaporated $11 \AA \mathrm{Pt}+14 \times(3.5 \AA \mathrm{Co}+11 \AA \mathrm{Pt})$ multilayer discussed primarily in this section, only small variations in the shape of the loops were observed. Both loops had $100 \%$ remanence and high nucleation fields. Larger differences existed, however, in the coercivities. The untreated disk had a coercivity of $154 \mathrm{kA} / \mathrm{m}$, while a simultaneously evaporated multilayer on an untreated glass substrate had a coercivity of $103 \mathrm{kA} / \mathrm{m}$. The coercivity of the sputter-etched disk was $102 \mathrm{kA} / \mathrm{m}$ and thus comparable to the coercivity of the $\mathrm{Co} / \mathrm{Pt}$ multilayer on glass.

The field dependence of the carrier and noise levels for these disks is shown in fig. 6. The carrier level of the sputter-etched disk showed a saturation at lower fields than for the untreated disk and the carrier level decreased more rapidly for fields in the erasure direction, i.e. sputter etching increased the field sensitivity. These effects were even more pronounced on disks with $14 \AA \mathrm{Pt}$ layers instead of $11 \AA$. The largest difference is however observed for the noise level. For the untreated disk the noise level is relatively high and nearly independent of the field. The sputter-etched disk has a

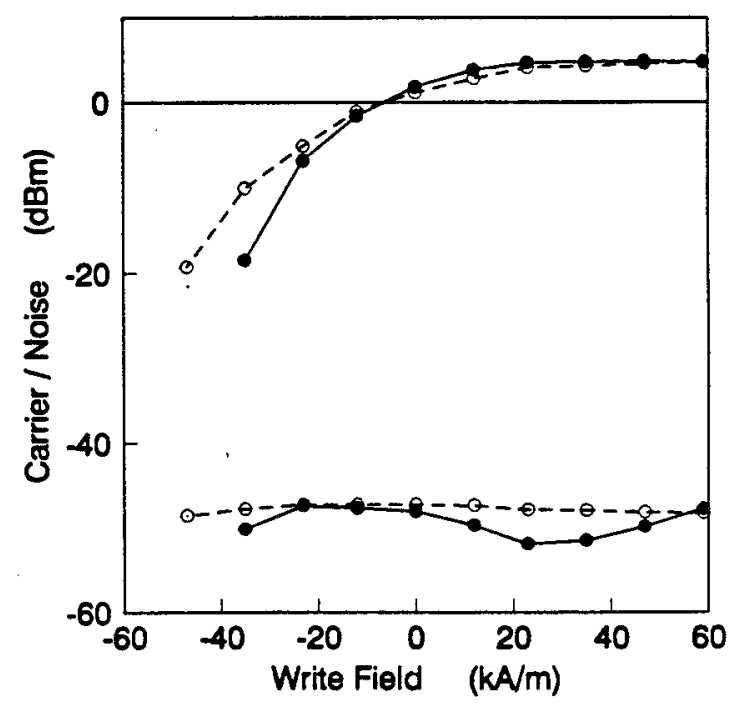

Fig. 6 Carrier and noise levels versus write field for an untreated disk with a $11 \AA \mathrm{Pt}+14 \mathrm{x}(3.5 \AA \mathrm{Co}+11 \AA \mathrm{Pt})$ multilayer (open circles) and a similar disk with a mildly sputter etched AlN layer (closed circles). Negative write fields are in the erasure direction. 
field-dependent noise level which is minimum for a write field of about $20 \mathrm{kA} / \mathrm{m}$ in the write direction. Also, the disk noise levels for the two disks were different. The sputter-etched disk had a $4 \mathrm{~dB}$ lower disk-noise level. These effects resulted in a maximum CNR of $53.1 \mathrm{~dB}$ at the highest applied field of $59 \mathrm{kA} / \mathrm{m}$ for the untreated disk and a $3.5 \mathrm{~dB}$ higher CNR (56.6 $\mathrm{dB})$ at $23 \mathrm{kA} / \mathrm{m}$ for the sputter-etched disk.

To find an explanation for these effects, the disks were imaged by SFM. Figs. 7a and b show MFM images of domains recorded on the untreated and on the sputter-etched disk, respectively. Fig. 7c is a SFM image of the surface structure of the untreated disk obtained by scanning with a non-magnetic tungsten tip. Besides the magnetic structure of the recorded domains, the MFM image of the sputter etched disk mainly shows the "smooth" surface structure of the tracks. Only a few small features can be seen on closer inspection within the grooves. On the other hand, the SFM image (fig. $7 \mathrm{c}$ ) of the untreated disk shows a rough structure which should be surface topography, because a non-magnetic tip was used. A similar rough surface structure is also seen in the MFM image of this disk (fig. 7a).

The rough microstructure of the untreated disk is most likely the origin of the increased coercivity as well as of the higher disk noise level. These effects are very similar to the effects observed for Pt base layers, which also enhance the coercivity as well as increase the disk noise level [11]. The more isolated multilayer grains on the rough base layer lead to a more "particulate" behaviour. This results in more sheared loops with higher coercivities. The disk noise originates either in the

\begin{tabular}{ll||c|c|c|c}
$\mathrm{H}_{\text {vr }}$ & $(\mathrm{kA} / \mathrm{m})$ & 60 & 30 & 0 & -30 \\
\hline $\mathrm{CNR}$ & $(\mathrm{dB})$ & 52.9 & 52.5 & 48.4 & 39.6 \\
\hline $\mathrm{C}$ & $(\mathrm{dBm})$ & 5.2 & 4.8 & 1.4 & -7.8 \\
\hline $\mathrm{WN}$ & $(\mathrm{dB})$ & 2.3 & 2.3 & 3.0 & 2.6
\end{tabular}

(a)

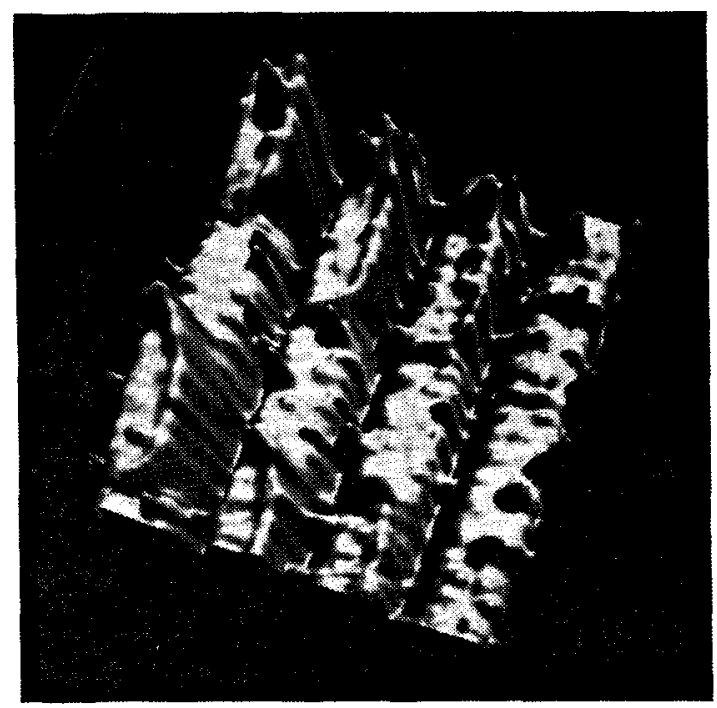

\begin{tabular}{ll||c|c|c|c}
$\mathrm{H}_{w r}$ & $(\mathrm{kA} / \mathrm{m})$ & 60 & 30 & 0 & -30 \\
\hline $\mathrm{CNR}$ & $(\mathrm{dB})$ & 51.9 & 56.8 & 49.3 & 34.5 \\
\hline $\mathrm{C}$ & $(\mathrm{dBm})$ & 4.9 & 5.0 & 1.8 & -14.6 \\
\hline $\mathrm{WN}$ & $(\mathrm{dB})$ & 8.0 & 3.2 & 7.5 & 5.9
\end{tabular}

(b)

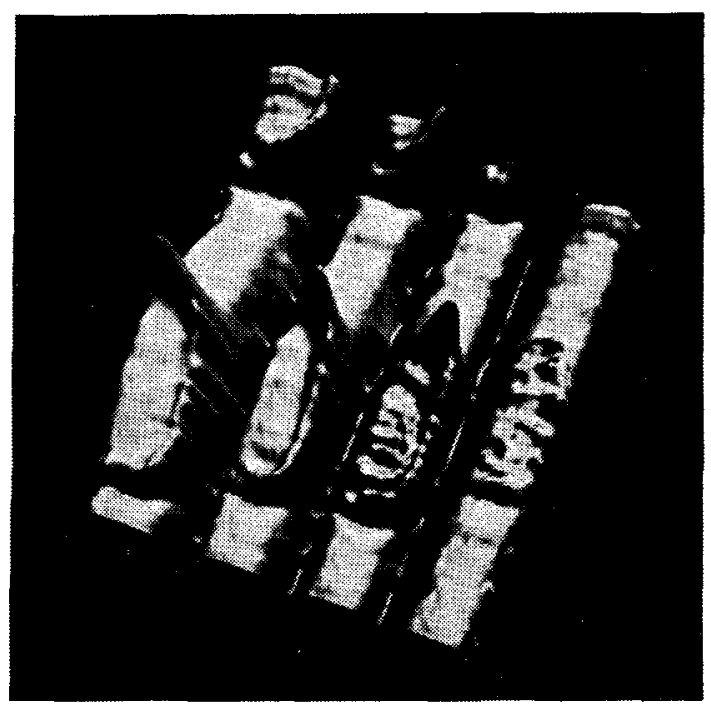

(c)

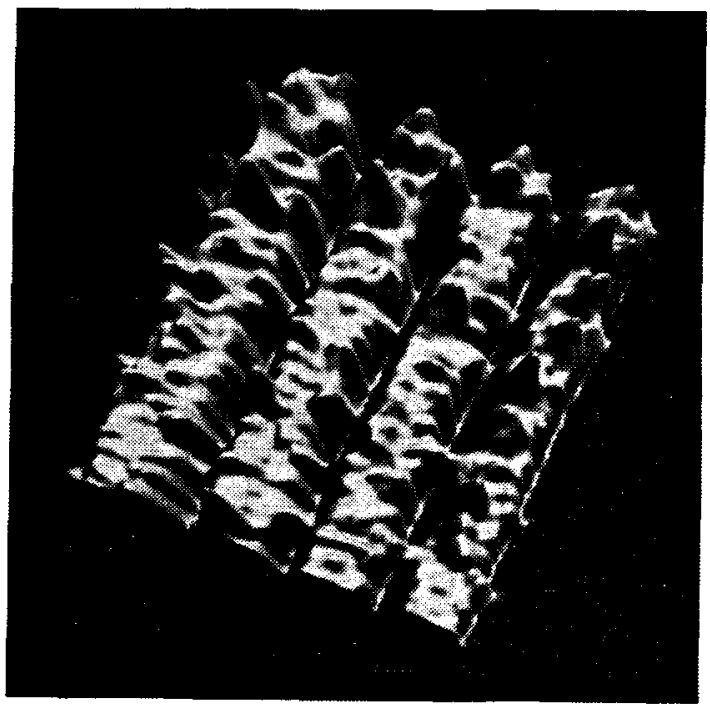

Fig. 7 MFM images of domains recorded on (a) an untreated and (b) a sputter-etched disk. Both disks have a $11 \AA \mathrm{Pt}+14 \times(3.5 \AA \mathrm{Co}$ $+11 \AA \mathrm{Pt}$ ) multilayer. The write fields as well as the CNR, carrier (C) and write noise (WN) of the read-out signal are given in the table. The order of the columns and the tracks is the same. Image (c) has been obtained by scanning over the surface of the untreated disk with a non-magnetic tip. 
reflectivity variations due to the roughness or in the variations in the Kerr rotation over the grains caused by the slightly different orientations.

The write noise was lower for the untreated disk than for the sputter-etched disk. The lower level for the disk with the rough surface may either be due to a masking by the high disk noise or to a domain boundary with less jaggedness. The latter might very well occur because the particulate layer possibly has a higher density of pinning sites, which prevent domain wall motion and thereby the occurrence of irregularities on the domain boundary. Comparison of the MFM images in fig. 7a and $b$ shows that no large differences exist in the jaggedness of the walls. Therefore, the increased write noise of the sputter-etched disk is most likely due to the fact that it is no longer masked by disk noise. The rough structure can provide as well the explanation for the slower saturation of the carrier level at high write fields. The high density of nucleation sites in the particulate layer leads to a greater tendency to form subdomains, which is difficult to suppress completely even in high write fields. From the MFM images in figs. $7 a$ and $b$ it is difficult, however, to draw detailed conclusions about the domain jaggedness and subdomain structure because of the dominance of the surface structure over the magnetic structure in fig. $7 \mathrm{a}$ and the resolution of "only" $100 \mathrm{~nm}$ in both images. The Co/Pt disk shown in fig. $2 \mathrm{a}$ also received a sputter-etch treatment and showed a similar recording behaviour as the sputter-etched disk described above. The resolution for this image is high enough to relate the small features in the magnetic structure to the field dependence of write noise as described in the first section.

\section{CONCLUSIONS}

The combination of a high resolution and essentially no sample preparation makes magnetic force microscopy a powerful characterization technique for magnetic storage media. This is illustrated by images of domains recorded on $\mathrm{GdTbFe}$ and $\mathrm{Co} / \mathrm{Pt} \mathrm{MO}$ disks. Of primary importance in MFM are the shape and magnetic structure of the tip. Inevitable imperfections in the microfabrication of the tip can lead to images with strong tip artefacts. Characterization of the tip by imaging a sample with a well-known structure is thus necessary before imaging a new sample.

SFM with magnetic and non-magnetic tips has been used to obtain more insight in the effects of a mild sputter etch of the dielectric layer before the deposition of a $\mathrm{Co} / \mathrm{Pt}$ multilayer. The sputter etch leads to smoother multilayers with less particulate character. This results in a lower disk noise and a higher field sensitivity. The latter is most likely related to a reduced number of nucleation and wall pinning centers which enables the suppression of subdomains at lower write fields.

\section{ACKNOWLEDGMENTS}

We would like to thank J.M. Kerkhof, H.C. Donkersloot, H. Wilting (PDO) and A.J. Mud for the deposition and characterization of the MO disks and $\mathrm{S}$. Porthun from the University of Twente for valuable discussions and assistance in the MFM experiments.

\section{REFERENCES}

[1] H.J. Mamin, D. Rugar, J.E. Stern, B.D. Terris, S.E. Lambert: Appl. Phys. Lett., 53,1563(1988).

[2] J.P.C. Bernards, A.J. den Boef: IEEE Trans. Magn., 26,1515(1990).

[3] J.P.J. Groenland, G.J.P. van Engelen, J.P.C. Bernards, H.A.J. Cramer: to be published in J. Magn. Magn. Mat., 120(1993).

[4] Y. Martin, D. Rugar, H.K. Wickramasinghe: Appl. Phys. Lett., 52,244(1988).

[5] H.W. van Kesteren, A.J. den Boef, W.B. Zeper, J.H.M. Spruit, B.A.J. Jacobs, P.F. Carcia: J. Magn. Soc. Jpn., 15 Suppl. S1,247(1991).

[6] H.W. van Kesteren, A.J. den Boef, W.B. Zeper, J.H.M. Spruit, B.A.J. Jacobs, P.F. Carcia: J. Appl. Phys., 70,2413(1991).

[7] W.B. Zeper, A.P.J. Jongenelis, B.A.J. Jacobs, H.W. van Kesteren: IEEE Trans. Magn., 28,2503(1992).

[8] A.J. den Boef: Appl. Phys. Lett., 56,2045(1990).

[9] P. Grütter, W. Zimmermann-Edling, D. Brodbeck: Appl. Phys. Lett., 60,2741(1992).

[10] P.F. Carcia, M. Reilly, W.B. Zeper, H.W. van Kesteren: Appl. Phys. Lett., 58,191(1991).

[11] W.B. Zeper, H.W. van Kesteren, B.A.J. Jacobs, J.H.M. Spruit, P.F. Carcia: J. Appl. Phys., 70,2264 (1991). 